\title{
The Cognitive Blending of Mathematics and Physics Knowledge
}

\author{
Thomas J. Bing and Edward F. Redish \\ Department of Physics, University of Maryland, College Park, MD 20742
}

\begin{abstract}
Numbers, variables, and equations are used differently in a physics class than in a pure mathematics class. In physics, these symbols not only obey formal mathematical rules but also carry physical ideas and relations. This paper focuses on modeling how this combination of physical and mathematical knowledge is constructed. The cognitive blending framework highlights both the different ways this combination can occur and the emergence of new insights and meaning that follows such a combination. After an introduction to the blending framework itself, several examples from undergraduate physics students' work are analyzed.
\end{abstract}

Keywords: cognitive modeling, blending, mathematics PACS: $01.30 .1 b, 01.40 . F k, 01.40 . \mathrm{Ha}$

\section{INTRODUCTION}

An important sign of physics students' progress is their combining the symbols and structures of mathematics with their physical knowledge and intuition, enhancing both. The numbers, variables, and equations of the mathematics come to represent physical ideas and relations. Likewise, physical intuitions become encoded in a precise way that readily lends itself to the complex manipulations often required of a physicist. New ideas and inferences emerge after this combination.

The language of cognitive blending provides a framework for analyzing students' combination of mathematics and physics. This framework emphasizes both the emergence of new relations and the different ways the combination itself can be constructed. Following an overview of the theory of cognitive blending, the framework will be applied to examples of physics students' work.

\section{THEORETICAL OVERVIEW}

The knowledge we have in long-term memory that we use to interpret our perceptions is organized into associational patterns, knowledge elements or resources that tend to be primed or activated together. See [1] and the references therein for a detailed discussion. Cognitive blending theory refers to these as mental spaces. Fauconnier and Turner [2,3] describe how the mind combines two or more mental spaces to make sense of linguistic input in new, emergent ways. Blending usually occurs at a subconscious level, although the explicit thought required in classroom activity causes many of its details to become explicitly apparent. Like the learning process itself, blending is nonlinear and nondeterministic. The precise way a person blends two input mental spaces together depends strongly on cues in the linguistic input and on physical and mental context.

\section{Example of a Blend}

Consider an example from outside of physics. If someone hears "Bill Gates knocked out Steve Jobs", that person will construct a meaning for the statement by blending two mental spaces. Mental spaces typically contain both elements and an organizing frame of relationships, processes, and transformations. A Boxing mental space containing elements such as opposing fighters, punches, injuries, and so on would be blended with a Business mental space containing elements like Gates, Jobs, price cuts, advertising campaigns, and profits. In the blended space, Boxing CEOs, statements like "Bill Gates knocked out Steve Jobs", "Jobs hurt Gates with a new advertising campaign", and "Gates landed a punch with his price cuts" make sense. Such statements can't occur in the Business space because no one is literally knocked out in business. Nor can such statements occur in the Boxing mental space; Gates and Jobs are not boxers. 
These statements are all examples of emergent meaning, ideas and relations that could not exist in either input mental space alone.

\section{Single-Scope And Double-Scope Blends}

Blends can be constructed in different ways. Fauconnier and Turner describe two types of blending topologies that are especially useful in considering students' combinations of physical and mathematical reasoning. These two types of blends are called single-scope and double-scope. Single-scope blends essentially only import elements from one input mental space into the organizing frame of the other. Double-scope blends display a blending of the organizing frames of the input mental spaces.

The earlier Boxing CEOs example can be diagrammed.

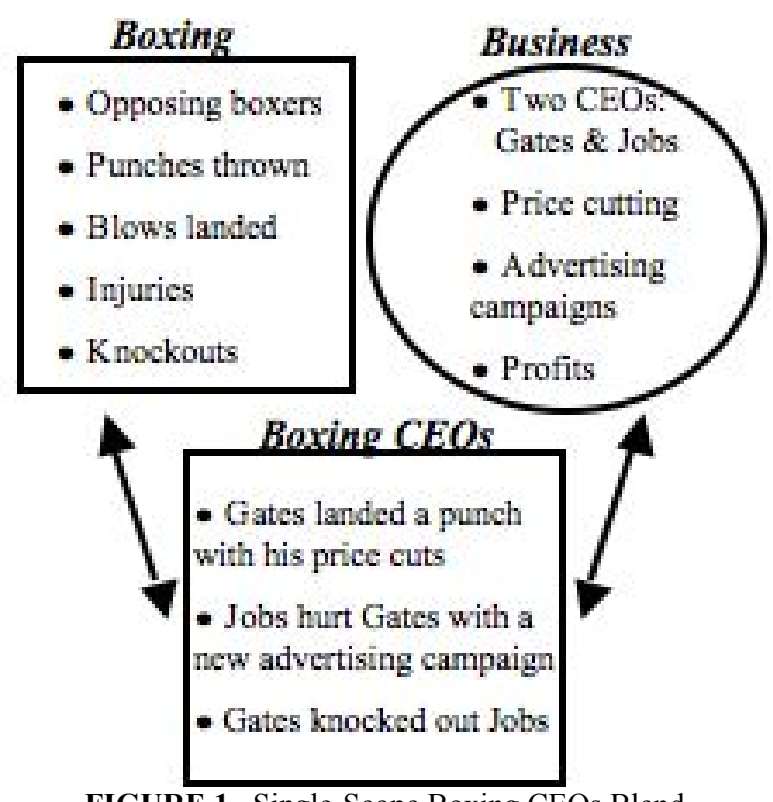

FIGURE 1. Single-Scope Boxing CEOs Blend

This blend is single-scope. The blended space's organizing structure is almost entirely that of the Boxing input mental space. To illustrate this point, consider the verbs used in the blended space. Landing a punch, hurting, and knocking out all come from the Boxing input space. The timescale of the blend is short and abrupt, like a boxing match, not drawn out over fiscal years. Combat in the blend is one-on-one and not corporation against corporation.

Just because a blend is single scope does not imply that no new insights are gained in the input space that only contributes elements to the blended space. On the contrary, most elements in a single-scope blended space inform the deemphasized input space upon projection back up through the blending network. All three of the elements in the Boxing CEOs space in Figure 1 ultimately convey a new idea or relation about business. The single-scope distinction arises from the structure of the blended space itself. That most of the emergent meaning of this blending network pertains to business is a consequence of the single-scope topology.

We claim that this sample blend is a common, reasonable way a person could construct meaning for a statement like "Bill Gates knocked out Steve Jobs" and not necessarily the only possible way. With the blending framework now illustrated, we continue to several examples from physics students' work. Here mathematical and physical mental spaces will be blended to produce emergent meaning.

\section{STUDENT BLENDING EXAMPLES}

The examples of student work analyzed here come from video tapes of physics students working outside of class on their homework. In addition to the video, the data library contains copies of the written work the students ultimately submitted.

\section{Double-Scope Air Drag Example}

This first example comes from a group of three physics majors enrolled in a Mathematical Methods in Physics class taught by the physics department, usually to students in their second or third year. They are working on a homework problem where an object is thrown straight up and falls back down under the forces of gravity and air resistance. In the following excerpts, they are working to understand the expression for the viscous force, $F_{V}=-b v$, as given in the homework problem. Their conversation includes

S1 Because the negative means that friction operates in the opposite direction of whatever $\mathrm{v}$ is

and

S2 Well, but let's do the first one first, if you're going down ... what is $\mathrm{v}$ gonna be? Is it gonna be negative or positive?

S3 It's gonna be negative.

S2 OK, so a negative times a negative

S3 Is gonna be positive

S2 Right, and a positive points up.

and

$\mathrm{S} 2 \mathrm{We}$ need to leave this negative in so that it cancels out that one 
S1 Right, because $\mathrm{v}$ has that negative built into it, and so we need another negative out here to make sure that the two negatives cancel out and you end up with a positive, which is up, which is the direction of friction because it's going down-it's falling down, being dragged in the upward direction

All of these excerpts come from the same two-minute clip. As before, a blending diagram can be drawn.

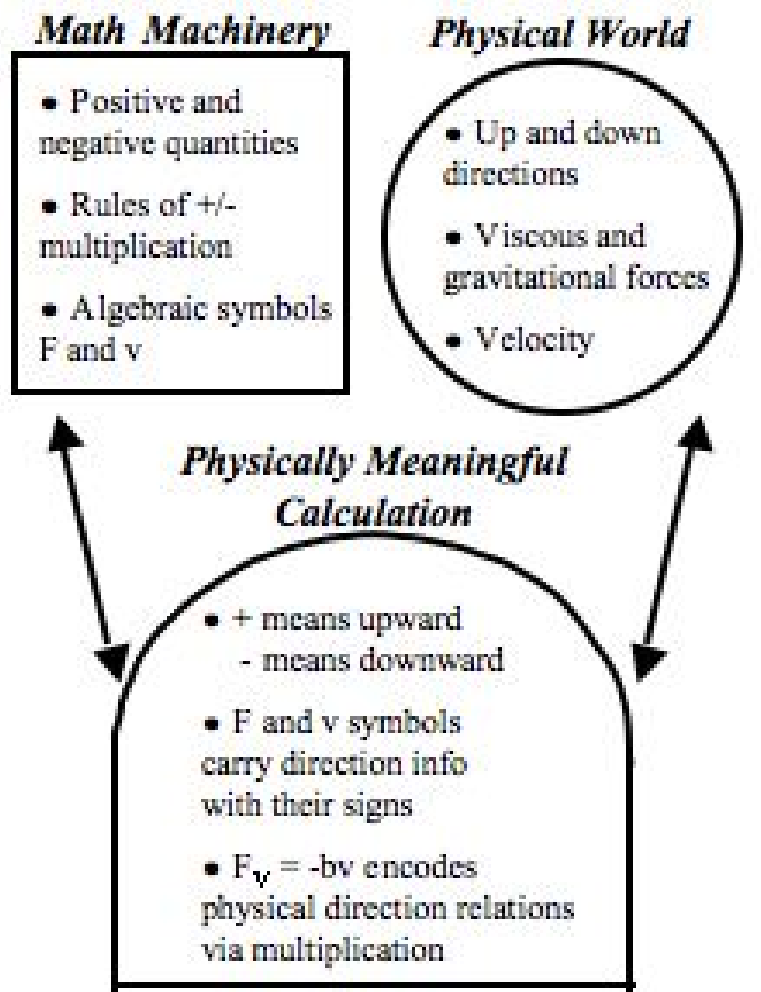

FIGURE 2. Double-Scope Air Drag Blend

This blend is double-scope. It represents a combination of organizing frames, not an importation of elements into the other input space's frame. Unlike the uni-directionality seen in the Boxing CEO example, the transcript examples above exhibit a bidirectionality. Sometimes the students start with a mathematical statement and translate it to a physical statement, as in the first excerpt. In the second excerpt, a physical statement is translated into a mathematical idea. Examining the role of positive and negative signs in this blend also indicates its doublescope nature. In the second and third excerpts these signs behave according to algebraic rules, but at the same time they encode physical information on the direction of the drag force that is carried along and expressed through these algebraic manipulations.
Whereas most of the emergent meaning that arose in the single-scope Boxing CEOs network pertained largely only to one input space, the situation is more balanced in the double-scope example. The third blended space element refines and codifies the physical observation that the air drag force must act opposite the direction of motion. At the same time, this compact expression will be used within a larger piece of mathematical machinery when the students insert it into Newton's Second Law and proceed to solve the resulting equation of motion.

The next example illustrates a single-scope blend of mathematics and physical reasoning. It shares the structural features of the Boxing CEOs example.

\section{Single-Scope Travel Time Example}

This example comes from a student enrolled in an algebra-based Introductory Mechanics course for biological science majors. The student is working with a teaching assistant on a homework problem asking how much time it would take a car traveling 95 feet per second to go 500 feet.

S4 So, I was trying to do a proportion, but that doesn't work. I was like 95 feet per second, oh wait, yeah in 500 feet, like, $x$ would be the time - that doesn't, I get like this huge number and that doesn't make any sense.

Having constructed the equation $95 / 1=x / 500$, the student has arrived at $\mathrm{x}=47,500$ seconds.

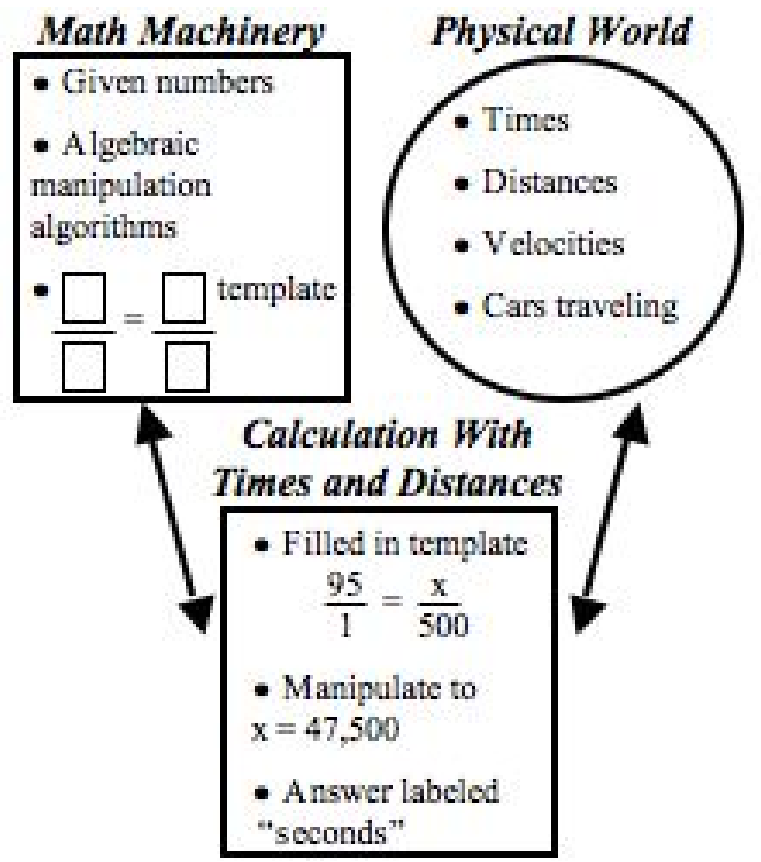

FIGURE 3. Single-Scope Travel Time Blend 
The student's work in the blended space is organized almost entirely by the Math Machinery input space's frame. Only elements like the label "seconds" that is tacked onto the final numeric answer come from the other input mental space, leading to the characterization of this blend as single-scope. Just as in the Boxing CEOs example, the emergent meaning of this network largely informs the deemphasized input space, in this case the Physical World mental space. The student interprets the final answer of 47,500 seconds as newly constructed information about how long the car requires to travel 500 feet. It is the unreasonableness encountered in this projection back up through the single-scope network that signals a problem to the student.

It is important to note that this student was quite capable of constructing a rich Physical mental space for the situation at hand. Immediately following the excerpt given above, the teaching assistant probes the student's physical intuitions in more detail.

TA That doesn't make any sense. So what if I said something like if you're traveling two feet per second and you go four feet. How long would that take you?

S4 two seconds

TA Or, if you tried different numbers, if I was traveling eight feet per second and you traveled sixteen feet, how long would that take you?

S4 two seconds

Both times the student answers quickly without formal, explicit calculation. The simple numbers the teaching assistant offered allowed the student to operate within a rich Physical mental space with excellent intuitions about rates of travel, distances, and times.

\section{DISCUSSION}

Use of the cognitive blending framework emphasizes the demands students face concerning the integration of their mathematical and physical knowledge. This integration process is complex and can be achieved in different ways, as the single-scope and double-scope distinction indicates. The difficulties experienced by the latter student stem not from a lack of prerequisite knowledge but from an inappropriate integration of two well established mental spaces.

In and of themselves, single-scope blends are not more or less appropriate than double-scope blends. The utility of a particular blending topology depends on the specific context. Double-scope blends of mathematical and physical mental spaces are more common in areas like mechanics and electricity and magnetism where a student's physical experiences and intuitions are more robust and available. It is quite possible that a more mathematically dominated topic like quantum mechanics would be marked by a heavier reliance on single-scope networks, even among experts.

Fauconnier and Turner note that the terms singlescope and double-scope are merely two convenient markers along a continuum of blending topologies. Observation of students' work with mathematics and physics, however, indicates that both types of blends have inertia. Students who initially approach a physics question with a heavily single-scope blend tend to continue working within that topology. Transition to a more double-scope blend of physical and mathematical knowledge often requires a strong perturbation to the system, such as a student suddenly considering a new piece of evidence or an instructor asking questions aimed at activating the deemphasized mental space. Awareness of this cognitive blending framework can help instructors more readily understand how students are thinking and offer appropriate guidance for the specific situation at hand. It can help researchers in providing a theoretical framework for description of student thought and perhaps even a structure for understanding what cues prompt students for blending in particular ways. Such a framework could potentially provide predictive power for development of effective instructional materials.

\section{ACKNOWLEDGMENTS}

The authors wish to thank David Hammer, Rachel Scherr, and the other members of the Physics Education Research Group at the University of Maryland for their insightful discussion. This work is supported by National Science Foundation grants DUE 05-24987 and REC 04-40113 and a Graduate Research Fellowship.

\section{REFERENCES}

1. E. F. Redish, "A Theoretical Framework for Physics Education Research: Modeling Student Thinking" in Proceedings of the Varenna Summer School, "Enrico Fermi" Course CLVI, Italian Physical Society, 2004.

2. G. Fauconnier and M. Turner, Cognitive Science 22(2), 133-187 (1998)

3. G. Fauconnier and M. Turner, The Way We Think: Conceptual Blending and the Mind's Hidden Complexities, New York: Perseus Books Group, 2002. 